\title{
Association between the histological subtype of lung adenocarcinoma, EGFR/KRAS mutation status and the ALK rearrangement according to the novel IASLC/ATS/ERS classification
}

\author{
YU-JIE DONG ${ }^{*}$, YI-RAN CAI ${ }^{*}$, LI-JUAN ZHOU, DAN SU, JING MU, XUE-JING CHEN and LI ZHANG \\ Department of Pathology, Beijing Chest Hospital, Capital Medical University, \\ Beijing Tuberculosis and Thoracic Tumor Research Institute, Beijing 101149, P.R. China
}

Received December 24, 2014; Accepted January 27, 2016

DOI: $10.3892 / \mathrm{ol} .2016 .4233$

\begin{abstract}
The present study aimed to investigate the association between epidermal growth factor receptor (EGFR)/Kirsten rat sarcoma viral oncogene homolog (KRAS) mutations, anaplastic lymphoma receptor tyrosine kinase (ALK) rearrangements and the morphological characteristics of lung adenocarcinoma (LAC), according to the International Association for the Study of Lung Cancer/American Thoracic Society/European Respiratory Society (IASLC/ATS/ERS) classification in a large group of patients with primary LAC. A total of 200 patients with invasive LAC who had undergone complete resections at the Beijing Chest Hospital (Beijing, China) were randomly selected. The morphology of the samples was reassessed in $5 \%$ increments by two pathologists, according to the IASLC/ATS/ERS scheme. EGFR and KRAS mutations were tested by direct DNA sequencing. ALK rearrangements were screened by immunohistochemistry on a Benchmark XT stainer. The data revealed that EGFR and KRAS mutations, and ALK rearrangements were identified in $46.0 \%$ (92/200), $9.0 \%$ (18/200) and $11.5 \%$ (23/200) of the patients, respectively. The EGFR/KRAS mutations and ALK rearrangements were mostly exclusive. However, 1 patient exhibited the coexistence of the EGFR (at exon 20) and KRAS (codon 12) mutations, and another patient exhibited the coexistence of the EGFR mutation (at exon 21) and the
\end{abstract}

Correspondence to: Dr Yi-Ran Cai, Department of Pathology, Beijing Chest Hospital, Capital Medical University, Beijing Tuberculosis and Thoracic Tumor Research Institute, 97 Machang Road, Tongzhou, Beijing 101149, P.R. China

E-mail: cyr_71@163.com

*Contributed equally

Key words: lung adenocarcinoma, mutation status, histological subtype, International Association for the Study of Lung Cancer/American Thoracic Society/European Respiratory Society
ALK gene fusion. EGFR mutations were indicated to be closely associated with the acinar predominant $(43 / 77 ; 55.8 \%$; $\mathrm{P}=0.030)$ and papillary predominant $(26 / 49 ; 53.1 \% ; \mathrm{P}=0.006)$ subtypes. KRAS mutations were more commonly associated with the solid predominant subtype $(9 / 52 ; 17.3 \% ; \mathrm{P}=0.023)$ and invasive mucinous $\operatorname{LAC}(5 / 10 ; 50.0 \% ; \mathrm{P}=0.004)$, and less commonly associated with the acinar predominant subtype $(1 / 77 ; 1.3 \%$; $\mathrm{P}=0.002)$. ALK rearrangements more commonly occurred in the solid predominant subtype compared with other subtypes $(13 / 52 ; 25 \% ; \mathrm{P}=0.002)$, and less commonly occurred in the papillary predominant subtype $(1 / 49 ; 2.0 \%$; $\mathrm{P}=0.004)$. Tumors harboring ALK rearrangements were characterized by signet-ring cell $(7 / 9 ; 77.8 \% ; \mathrm{P}<0.0001)$ and cribriform $(7 / 12 ; 58.3 \%$; $\mathrm{P}<0.0001)$ patterns. The association between the mutation status and histological subtype in LAC was distinct. The predominant subtype according to the IASLC/ATS/ERS classification provided important information for gene mutations and integrated clinical findings to improve the treatment of LAC patients.

\section{Introduction}

Lung adenocarcinoma (LAC) is a major histological subtype of lung cancer (1) and a heterogeneous tumor that includes various histological subtypes, molecular alterations and responses to chemotherapy and targeted therapy (2). Patients with LAC have benefited less from traditional chemotherapy (3). With the increasing administration of targeted therapy, the driver genes harbored in LAC may receive increased consideration. Targeted therapy has been associated with great achievements towards improving the clinical outcomes of LAC patients (4). For example, targeted therapy has been shown to increase the median progression-free survival time of patients when compared with standard chemotherapy (5). The epidermal growth factor receptor (EGFR) and Kristen rat sarcoma viral oncogene homolog (KRAS) mutations have been reported as the major driver genes in LAC; the mutations are expressed exclusively and are characterized by distinct histological subtypes $(6,7)$. EGFR mutations have been verified as good independent prognostic factors for patients who are treated with gefitinib and erlotinib therapy (8). LAC with KRAS mutations 
demonstrates resistance to EGFR-targeted therapies. Therefore, KRAS and EGFR mutations have been screened together for the routine molecular testing of LAC (9). The anaplastic lymphoma receptor tyrosine kinase (ALK) rearrangement was first detected in LAC by Soda et al (10) in 2007. The detection of this rearrangement was a revolutionary point in the history of molecular therapy for LAC, and crizotinib as a specific targeted drug has obtained encouraging outcomes with regard to improving the survival of patients who harbor ALK rearrangements (11).

Increasing numbers of studies have focused on the histological subtypes of LAC following the novel classification proposed by the International Association for the Study of Lung Cancer/American Thoracic Society/European Respiratory Society (IASLC/ATS/ERS) $(12,13)$. Finding the association between the histological subtype and mutation status of LAC patients is crucial. Numerous previous studies have evaluated the association between EGFR/KRAS mutation status, ALK rearrangement and histological subtype (14-17). However, the results of these studies have been controversial. EGFR mutations have most often been examined in papillary and micropapillary subtypes, and KRAS mutations in solid subtypes (6). Yet, Yoshizawa et al (14) reported that EGFR mutations were significantly associated with lepidic and papillary subtypes, and KRAS mutations with adenocarcinomas with mucin production. In addition, a previous study confirmed that a solid signet-ring cell pattern and a mucinous cribriform pattern were the most common histological features in ALK-positive tumors (16), whereas another study revealed a significant association between intra- and/or extra-cytoplasmic mucin and cribriform patterns in tumors with ALK rearrangements (17).

Following the established IASLC/ATS/ERS classification of LAC, the purpose of the present study was to identify the associations between EGFR/KRAS mutation alterations and ALK rearrangements and the predominant subtypes in 200 LAC patients.

\section{Patients and methods}

Patients. Cases of invasive LAC in which the patients had undergone surgery at the Beijing Chest Hospital (Beijing, China) between March 2005 and June 2014 were randomly selected from the hospital medical archives. Clinical data were collected, including gender, age, smoking status, tumor size, lymph node metastases and stage. The use of archived samples and the protocols in the present study were approved by the Medical Ethics Committee of Beijing Chest Hospital, Capital Medical University (approval no., 2014-No.10).

'Never smokers' were defined as patients who had smoked $<100$ cigarettes in their lifetime. The patients were not provided any treatment prior to surgery. Tumor staging was decided according to the 7th edition of the American Joint Committee for Cancer staging system (18). The morphology of all samples was reviewed by at least two pathologists, according to the 2011 IASLC/ATS/ERS international multidisciplinary classification of LAC (19).

Tumor samples. Formalin-fixed, paraffin-embedded (FFPE) samples, stained with hematoxylin and eosin, were reviewed by two pathologists. FFPE blocks were divided into 5-10 sections of 5- $\mu \mathrm{m}$ thickness. EGFR and KRAS gene mutations were screened using the direct DNA sequencing method (20). Mutations in exons 18, 19, 20 and 21 of EGFR and codons 12 and 13 of KRAS mutations were detected in the present study.

$D N A$ extraction, polymerase chain reaction $(P C R)$ amplification and direct sequencing for EGFR/KRAS mutations. FFPE blocks were divided into $10-154-\mu \mathrm{m}$ thick sections for DNA extraction according to the previously described protocol (21). Briefly, PCR was performed with $100 \mathrm{ng}$ template DNA in a $50-\mu 1$ volume that contained 0.75 units of HotStarTaq DNA polymerase (Fermentas International Inc.; Thermo Fisher Scientific, Waltham, MA, USA), $5 \mu \mathrm{l}$ PCR buffer, $0.8 \mu \mathrm{M}$ deoxyribonucleotide triphosphate, $0.5 \mu \mathrm{M}$ of each primer and various concentrations of $\mathrm{MgCl}_{2}$, depending on the various markers. PCR was performed on a Genepro Thermal Cycler (TC-E-96G; Hangzhou Bioer Technology Co., Ltd., Hangzhou, China) and the cycling conditions were as follows: 40 cycles of a denaturation step at $94^{\circ} \mathrm{C}$ for $45 \mathrm{sec}$, a primer annealing step at $56^{\circ} \mathrm{C}$ for $30 \mathrm{sec}$ and an elongation step at $72^{\circ} \mathrm{C}$ for $30 \mathrm{sec}$; and a final extension step at $72^{\circ} \mathrm{C}$ for $10 \mathrm{~min}$. The nucleic acid used for mutations was based on the reference sequence NM_005228.3 (www.ncbi.nlm.nih.gov/gene/? term=NM_005228.3). The primers (Sangon Biotech Co., Ltd., Shanghai, China) were designed for EGFR/KRAS mutations using Primer 3 software (primer3.ut.ee/) as follows: EGFR mutation: Exon 18 forward, 5'-CAACCAAGCTCTCTTGAG GATC-3' and reverse, 5'-CCCAGCCCAGAGGCCTGT-3'; exon 19 forward, 5'-GCAGCATGTGGCACCATCTC-3' and reverse, 5'-AGAGCCATGGACCCCCACAC-3'; exon 20 forward, 5'-CACACTGACGTGCCTCTCC-3' and reverse, 5'-AGCAGGTACTGGGAGCCAAT-3'; and exon 21 forward, 5'-TCTGTCCCTCACAGCAGGGTCT-3' and reverse, 5'-GCT GGCTGACCTAAAGCCACC-3'. $\beta$-actin was used as positive control: forward, 5'AGAGATGGCCACGGCTGCTT-3' and reverse, 5'-ATTTGCGGTGGACGATGGAG-3'. The primer sequence for the amplification of KRAS 12 and 13 codons mutation test referred to the study by Franklin et al (22) as follows: Forward, 5'-AAGGCCTGCTGAAAATGAC-3' and reverse, 5'-TGGTCCTGCACCAGTAATATG-3'. The analysis was performed according to the manufacturer's protocols using an ABI PRISM 377 DNA Sequencer (Applied Biosystems, Inc.; Thermo Fisher Scientific).

The detection of the ALK rearrangement was performed using Ventana immunohistochemistry on a Benchmark XT autostainer (Ventana Medical Systems, Inc., Tucson, AZ, USA), and with monoclonal rabbit anti-human ALK antibody (ready to use; clone, D5F3; catalog no., 790-4794; Ventana Medical Systems, Inc.). The Optiview DAB IHC detection kit (catalog no., 860-099; Ventana Medical Systems, Inc.) was used according to the manufacturer's protocols. Positive staining was indicated by strong granular staining in the tumor cell cytoplasm (Fig. 1A), in any percentage of positive tumor cells; otherwise, the expression of ALK was considered to be negative (23).

Statistical analysis. The associations between EGFR/KRAS mutation status and ALK rearrangement, clinical factors and 
Table I. Clinicopathological characteristics of EGFR/KRAS mutation status and ALK rearrangement in 200 patients.

\begin{tabular}{|c|c|c|c|c|c|c|c|c|c|}
\hline \multirow[b]{2}{*}{ Clinical features } & \multicolumn{3}{|c|}{ EGFR } & \multicolumn{3}{|c|}{ KRAS } & \multicolumn{3}{|c|}{ ALK rearrangement } \\
\hline & Mutation & WT & P-value & Mutation & WT & P-value & Positive & Negative & P-value \\
\hline Gender & & & 0.016 & & & 0.323 & & & 0.663 \\
\hline Male & 35 & 60 & & 11 & 84 & & 12 & 83 & \\
\hline Female & 57 & 48 & & 7 & 98 & & 11 & 94 & \\
\hline Age, years & & & 0.478 & & & 0.089 & & & 0.001 \\
\hline Range & $28-76$ & $23-79$ & & $50-78$ & $23-79$ & & $23-69$ & $28-79$ & \\
\hline Median & 59 & 57 & & 63 & 58 & & 47 & 61 & \\
\hline$<60$ & 46 & 60 & & 6 & 100 & & 20 & 86 & \\
\hline$\geq 60$ & 46 & 48 & & 12 & 82 & & 3 & 91 & \\
\hline Smoking status & & & 0.542 & & & 0.429 & & & 0.810 \\
\hline Yes & 26 & 35 & & 7 & 54 & & 6 & 55 & \\
\hline No & 66 & 73 & & 11 & 128 & & 17 & 122 & \\
\hline Tumor size, $\mathrm{cm}$ & & & 0.139 & & & 0.001 & & & 0.564 \\
\hline$\leq 5$ & 80 & 85 & & 9 & 156 & & 18 & 147 & \\
\hline$>5$ & 12 & 23 & & 9 & 26 & & 5 & 30 & \\
\hline LN metastasis & & & 0.257 & & & 0.622 & & & 0.046 \\
\hline Yes & 45 & 62 & & 11 & 96 & & 17 & 90 & \\
\hline No & 47 & 46 & & 7 & 86 & & 6 & 87 & \\
\hline Clinical stage & & & 1.000 & & & 1.000 & & & 0.076 \\
\hline $\mathrm{I}+\mathrm{II}$ & 45 & 53 & & 9 & 89 & & 7 & 91 & \\
\hline III+IV & 47 & 55 & & 9 & 93 & & 16 & 86 & \\
\hline
\end{tabular}

EGFR, epidermal growth factor receptor; KRAS, Kirsten rat sarcoma viral oncogene homolog; ALK, anaplastic lymphoma receptor tyrosine kinase; LN, lymph node.

the predominant subtype were compared by Fisher's exact test. All statistical tests were performed on SPSS version 21.0 (IBM SPSS, Armonk, NY, USA), and values of $\mathrm{P}<0.05$ for the two-tailed test were considered to indicate a statistically significant difference.

\section{Results}

Patient characteristics. Out of 200 patients, 95 (47.5\%) were male. The median age was 59 years (range, 23-79 years) and 61 patients had a history of smoking. All patients were diagnosed at various clinical stages: 55 patients at stage IA, 23 patients at stage IB, 17 patients at stage IIA, 3 patients at stage IIB, 80 patients at stage IIIA, 13 patients at stage IIIB and 9 patients at stage IV. The tissue sections were confirmed to possess enough tumor cells for EGFR and KRAS mutation and ALK fusion gene assays. The clinical data, including gender, age, smoking status, tumor size, lymph node metastasis and clinical stage, were reviewed. The clinicopathological features are summarized in Table I.

Histological features. According to the IASLC/ATS/ERS classification, the patients were grouped into the following subtypes: Lepidic predominant, 5 patients $(2.5 \%)$; acinar predominant, 77 patients $(38.5 \%)$; solid predominant with mucin production, 52 patients (26.0\%); papillary predominant, 49 patients $(24.5 \%)$; invasive mucinous adenocarcinoma, 10 patients (5.0\%); micropapillary predominant, 7 patients $(3.5 \%)$; lepidic component, 51 patients $(25.5 \%)$; intra-/extra-cytoplasmic mucus, 47 cases (23.5\%); cribriform pattern, 12 patients $(6.0 \%)$; and signet-ring cell, 9 patients $(4.5 \%)$ (Fig. 1B-F).

Associations between EGFR/KRAS mutations and ALK rearrangements and the predominant morphological structure EGFR mutations. EGFR mutations were examined in 92 patients (46\%), including 6 cases with mutations in exon 18 , 42 cases in exon 19, 6 cases in exon 20 and 32 cases in exon 21. The remaining 6 patients exhibited multiple mutations, of which 3 patients possessed sensitive mutations in exons 18 , 19 or 21 ; and 3 patients possessed sensitive and primarily resistant mutations in the exon 20 insertion. The frequency of EGFR mutation was increased in females compared with males $(\mathrm{P}=0.016)$.

The predominant subtype in 92 EGFR-mutant tumors was as follows: $3(60.0 \%)$ lepidic predominant, $43(55.8 \%)$ acinar predominant, $26(53.1 \%)$ papillary predominant, $49(57.1 \%)$ acinar predominant, 15 (28.8\%) solid predominant with mucin production and 1 (10\%) invasive mucinous adenocarcinoma.

EGFR mutations were more common in patients with the acinar predominant subtype $(43 / 77 ; 55.8 \% ; \mathrm{P}=0.030)$ and the papillary predominant subtype $(26 / 49 ; 53.1 \%$; $\mathrm{P}=0.006)$. Compared with the solid predominant subtype with mucin 
Table II. Correlation between EGFR/KRAS mutation status and ALK rearrangement with the histological subtype.

\begin{tabular}{|c|c|c|c|c|c|c|c|c|c|}
\hline \multirow[b]{2}{*}{ Predominant subtype } & \multicolumn{3}{|c|}{ EGFR } & \multicolumn{3}{|c|}{ KRAS } & \multicolumn{3}{|c|}{ ALK rearrangement } \\
\hline & Mutation & WT & P-value & Mutation & WT & P-value & Positive & Negative & P-value \\
\hline \multicolumn{10}{|l|}{ Lepidic predominant } \\
\hline Yes & 3 & 0 & & 0 & 0 & & 0 & 0 & \\
\hline No & 89 & 108 & & 18 & 182 & & 23 & 177 & \\
\hline \multicolumn{10}{|l|}{ Acinar predominant } \\
\hline Yes & 43 & 34 & 0.030 & 1 & 76 & 0.002 & 6 & 71 & 0.256 \\
\hline No & 49 & 74 & & 17 & 106 & & 17 & 106 & \\
\hline \multicolumn{10}{|l|}{ Papillary predominant } \\
\hline Yes & 26 & 23 & 0.038 & 3 & 46 & 0.120 & 1 & 48 & 0.004 \\
\hline No & 66 & 85 & & 15 & 136 & & 22 & 129 & \\
\hline \multicolumn{10}{|c|}{ Micropapillary predominant } \\
\hline Yes & 4 & 3 & & 0 & 7 & & 2 & 5 & \\
\hline No & 88 & 105 & & 18 & 175 & & 21 & 172 & \\
\hline \multicolumn{10}{|l|}{ Solid predominant } \\
\hline Yes & 15 & 37 & 0.006 & 9 & 43 & 0.023 & 13 & 39 & 0.002 \\
\hline No & 77 & 71 & & 9 & 139 & & 10 & 138 & \\
\hline \multicolumn{10}{|c|}{ Invasive mucinous adenocarcinoma } \\
\hline Yes & 1 & 9 & 0.040 & 5 & 5 & 0.004 & 1 & 9 & 1.000 \\
\hline No & 91 & 99 & & 13 & 177 & & 22 & 168 & \\
\hline
\end{tabular}

${ }^{a}$ The predominant subtype according the International Association for the Study of Lung Cancer/American Thoracic Society/European Respiratory Society classification. Only patients with acinar predominant, papillary predominant, solid predominant with mucin production and invasive mucinous adenocarcinoma were analyzed, since cases with lepidic and micropapillary predominant subtypes were too few (5 and 7 cases, respectively. EGFR, epidermal growth factor receptor; KRAS, Kirsten rat sarcoma viral oncogene homolog; ALK, anaplastic lymphoma receptor tyrosine kinase; WT, wild-type.

production $(15 / 52 ; 28.8 \% ; \mathrm{P}=0.004)$ and invasive mucinous adenocarcinoma $(1 / 10 ; 10.0 \% ; \mathrm{P}=0.007)$, EGFR mutations were more common in patients with the acinar predominant subtype $(43 / 77,55.8 \%)$. The papillary predominant subtype (26/49; 53.1\%) was more frequently detected in EGFR-mutant patients compared with patients with the solid predominant subtype with mucin production $(15 / 52 ; 28.8 \%$; $\mathrm{P}=0.016)$ and invasive mucinous adenocarcinoma $(1 / 10 ; 10.0 \% ; \mathrm{P}=0.016)$. EGFR mutations were less commonly identified in adenocarcinoma with mucin production $(11 / 47 ; 23.4 \%$; $\mathrm{P}=0.001)$ (Table II).

KRAS mutations. In total, 18 patients $(9.0 \%)$ harbored KRAS mutations, and 17 tumors were confirmed to contain KRAS mutations within codon 12 and 1 tumor contained KRAS mutations in codon 13 . KRAS mutations were significantly associated with tumor size $(\mathrm{P}=0.001)$.

KRAS mutations were distributed in $1(1.3 \%)$ patient with the acinar predominant subtype, $3(6.1 \%)$ with the papillary predominant, $9(17.3 \%)$ with the solid predominant subtype with mucin production, and $5(50 \%)$ with invasive mucinous adenocarcinomas. No KRAS mutation was identified in the lepidic and micropapillary predominant subtypes.

KRAS mutations occurred most frequently in the solid predominant subtype $(9 / 52 ; 17.3 \% ; \mathrm{P}=0.023)$ and the invasive mucinous adenocarcinoma subtype $(5 / 10 ; 50.0 \% ; \mathrm{P}=0.004)$, and less frequently occurred in the acinar predominant subtype $(1 / 77 ; 1.3 \% ; \mathrm{P}=0.002)$ and lepidic component $(1 / 50$; $2.0 \% ; \mathrm{P}=0.047)$. KRAS mutations were more common in the invasive mucinous subtype $(5 / 10 ; 50.0 \%)$ compared with acinar predominant tumors $(1 / 77 ; 1.3 \% ; \mathrm{P}<0.001)$ and the papillary predominant subtype $(3 / 49 ; 6.1 \% ; \mathrm{P}=0.002)$. KRAS mutations were also characterized by adenocarcinoma with mucin production $(9 / 47 ; 19.1 \% ; \mathrm{P}=0.008)$ (Table II).

ALK rearrangements. Among the 200 patients, ALK rearrangements were screened in 23 patients $(11.5 \%)$. The predominant subtypes in tumors harboring ALK rearrangements were as follows: Acinar predominant, 6 patients (7.8\%); lepidic predominant, 0 patients $(0.0 \%)$; papillary predominant, 1 patient (2.0\%); micropapillary predominant, 2 patients (28.6\%); solid predominant subtype with mucin production, 13 patients $(25.0 \%)$; and invasive mucinous adenocarcinoma, 1 patient (10.0\%). The ALK fusion gene was significantly associated with young age $(\mathrm{P}=0.001)$ and lymph node metastasis $(\mathrm{P}=0.046)$.

ALK rearrangements occurred most often in patients with the solid predominant subtype with mucin production $(13 / 52$; $25.0 \% ; \mathrm{P}=0.002$ ) and were less common in the papillary predominant subtype $(1 / 49 ; 2.0 \% ; \mathrm{P}=0.004)$. ALK-positive tumors occurred more frequently in the solid predominant subtype with mucin production $(13 / 52 ; 25 \%)$ compared with 

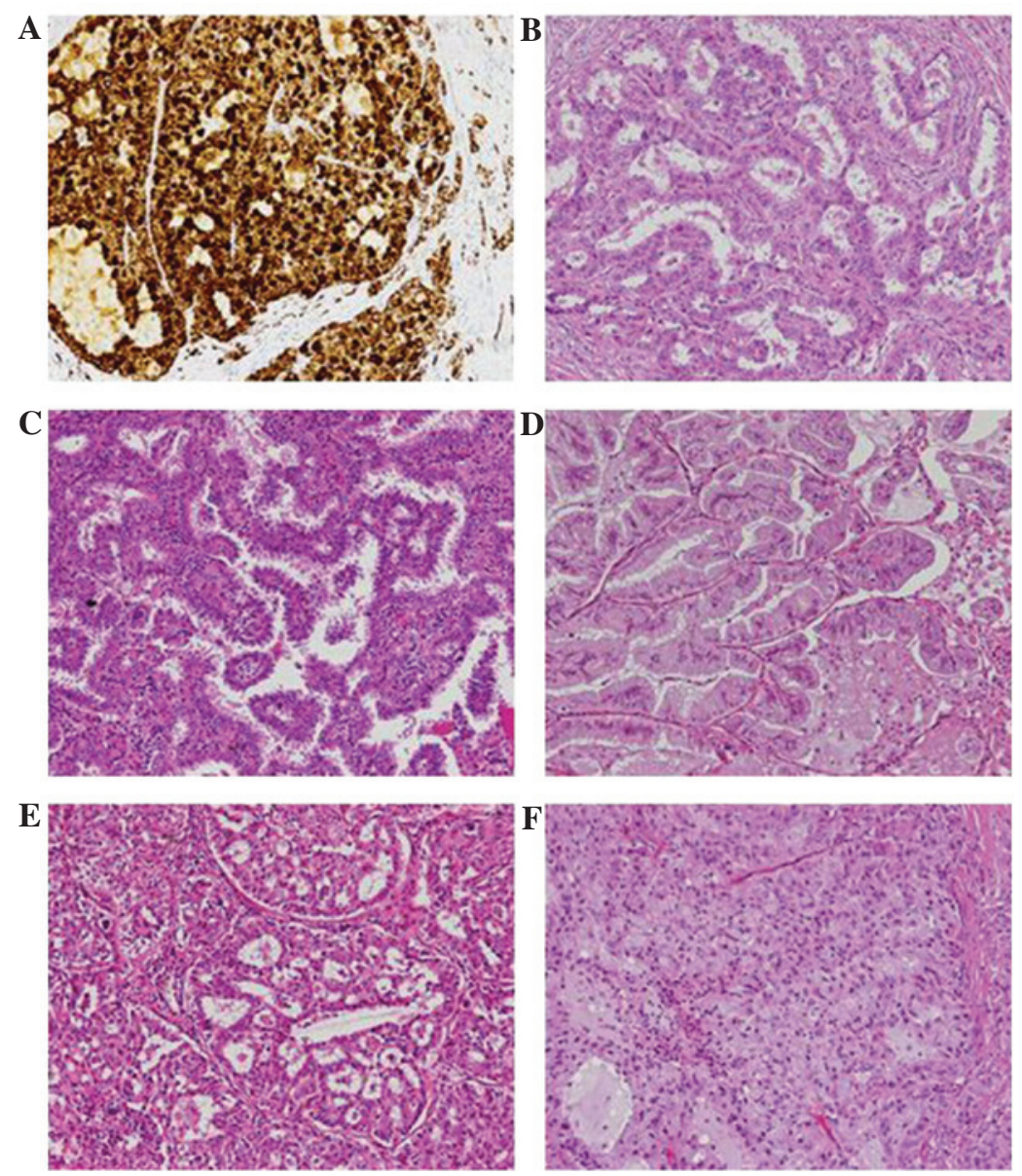

Figure 1. Histopathological characteristics. (A) ALK-positive tumors showing strong granular staining in the tumor cytoplasm, as determined using Ventana anti-ALK staining (magnification, x200). Growth patterns of lung adenocarcinoma were demonstrated using hematoxylin-eosin staining in (B-F). (B) Acinar predominant adenocarcinoma, (C) papillary predominant adenocarcinoma, (D) invasive mucinous adenocarcinoma, (E) acinar predominant adenocarcinoma showing components of a cribriform structure, and (F) solid predominant with mucin production adenocarcinoma showing components of a signet-ring cell pattern (magnification, x200).

the acinar predominant $(6 / 77 ; 7.8 \% ; \mathrm{P}=0.010)$ and papillary predominant $(1 / 49 ; 2.0 \% ; \mathrm{P}=0.001)$ subtypes. ALK-positive tumors had a tendency to express a characteristic morphological pattern, including solid predominant subtype with mucin production $(13 / 52 ; 25.0 \%)$, cribriform structure $(7 / 12$; $58.3 \% ; \mathrm{P}<0.001)$, intra-/extra-cytoplasmic mucin $(14 / 47$; $29.8 \% ; \mathrm{P}<0.001)$ and signet-ring cell presence $(7 / 9 ; 77.8 \%$; $\mathrm{P}<0.0001$ ) (Table II).

Coexistence of EGFR/KRAS mutation status and ALK rearrangement. Only 1 sample possessed the EGFR mutation (L858R) and ALK rearrangement, and another possessed an insertion mutation in exon 20 of the EGFR gene and a missense mutation in codon 12 of the KRAS gene.

\section{Discussion}

Prior to the establishment of the novel IASLC/ATS/ERS LAC classification, certain studies demonstrated the association between EGFR/KRAS mutation status or ALK rearrangement and adenocarcinoma histology according to the 2004 World Health Organization classification $(6,16)$. Motoi et al $(6)$ reported a significant association between the papillary and micropapillary LAC subtypes and the EGFR mutation.
Although several previous studies have identified the significant associations of the driver gene mutations and the predominant subtypes according to the proposed IASLC/ATS/ERS LAC classification $(24,25)$, the association between the genotype and subtype of LAC remains unclear. Numerous studies have identified the associations between EGFR mutations and the predominant subtypes subsequent to the publication of the novel IASLC/ATS/ERS classification. Wang et al (26) examined 332 patients with primary LAC, and 149 patients (44.9\%) were found to harbor EGFR mutations; the EGFR mutation rate was significantly increased in the papillary predominant subtype compared with the solid predominant subtype with mucin production $(\mathrm{P}=0.008)$. Song et al (24) reviewed 161 patients who underwent surgical resections, and EGFR mutation was examined in 67 cases $(41.6 \%)$. The EGFR mutation was identified more frequently in the micropapillary predominant $(\mathrm{P}=0.0068)$ and lepidic component $(\mathrm{P}=0.005)$ subtypes. The study by Yoshizawa et al $(14)$, which retrospectively examined 167 Japanese patients, indicated that the EGFR mutation was observed in 90 patients (53.9\%), and demonstrated a significant association with the adenocarcinomas with non-mucinous lepidic $(\mathrm{P}<0.0001)$ and papillary components $(\mathrm{P}<0.0001)$. Zhang et al $(27)$ enrolled 349 female patients who were never-smokers and identified EGFR 
mutations in 266 patients $(76.2 \%)$; the predominant subtype was the acinar predominant subtype $(\mathrm{P}=0.005)$.

The 4 aforementioned previous studies from Asia revealed that EGFR mutations were characterized by the acinar, papillary and micropapillary subtypes, and non-mucinous lepidic growth. In the present study, EGFR mutations were more common in the acinar predominant subtype $(\mathrm{P}=0.030)$ and papillary predominant subtype $(\mathrm{P}=0.006)$, and were rarely examined in adenocarcinomas with mucin production $(\mathrm{P}=0.001)$. The present findings were consistent with the studies by Yoshizawa et al (14) and Zhang et al (27). However, the present study did not find any significant association between EGFR mutations and the micropapillary and lepidic predominant subtypes, due to the small sample size for each ( $n=7$ and $n=5$, respectively).

The significant association noted between KRAS mutations and histological subtypes is controversial. Wang et al (26) did not identify any significant differences in histological subtypes between the KRAS mutations and the wild-types; whereas, Yoshizawa et al (14) and Kakegawa et al (28) indicated that KRAS mutations were significantly associated with adenocarcinoma of mucinous tumor subtypes $(\mathrm{P}<0.001)$. Rekhtman et al $(29)$ showed that KRAS mutations occurred more often in adenocarcinomas with a solid growth pattern $(\mathrm{P}=0.022)$.

The present findings indicated that KRAS mutations were characterized by the solid predominant subtype with mucin production $(\mathrm{P}=0.023)$ and invasive mucinous adenocarcinoma $(\mathrm{P}=0.004)$, and were also more frequently identified in LAC with mucin production $(\mathrm{P}=0.008)$. However, KRAS mutations were less frequently indicated in the acinar predominant subtype $(\mathrm{P}=0.002)$ and adenocarcinomas with lepidic component $(\mathrm{P}=0.047)$, which was in consistent with the report by Rekhtman et al (29).

Based on the IASLC/ATS/ERS classification of LAC, several studies $(15,16)$ have confirmed that the acinar predominant subtype, solid growth pattern, cribriform structure, mucin production and lack of lepidic growth were more common in ALK-positive patients. In the study by Yoshida et al (16), a solid signet-ring cell pattern and a mucinous cribriform pattern were examined in 43 and $56 \%$ of ALK-positive cases, respectively. Jokoji et al (17) indicated that ALK-positive tumors showed significant associations with intra-/extra-cytoplasmic mucin $(\mathrm{P}=0.0001)$, and the cribriform pattern with excessive extracytoplasmic mucin $(\mathrm{P}<0.0001)$.

Cribriform structure, as a unique pattern of the acinar subtype, was identified for being associated with the distinct histopathological features of ALK rearrangement in a previous study (30). A significant association between ALK rearrangement and cribriform structure $(\mathrm{P}<0.0001)$ was also indicated in the present study. ALK rearrangements occurred most often in the solid predominant subtype with mucin production $(\mathrm{P}=0.002)$ and less frequently in the papillary predominant subtype $(\mathrm{P}=0.004)$. ALK-positive tumors were also characterized by signet-ring cell presence $(\mathrm{P}<0.001)$ and adenocarcinoma with mucin production $(\mathrm{P}=0.0001)$.

The EGFR/KRAS mutation status and ALK rearrangement were mutually exclusive with each other (31). However, several studies $(26,32)$ have reported the coexistence of two mutations of the three driver genes. In the present study, 1 tumor possessed the
EGFR mutation (L858R at exon 21) and ALK rearrangement, and 1 tumor possessed the EGFR mutation (at exon 20) and KRAS mutation (codon 12). The KRAS mutation and the ALK rearrangement did not occur together in the sample of patients, and no cases exhibited all three driver gene mutations. The coexistence of the two genes is rarely reported. Determination of an effective treatment for patients who exhibit the coexistence of gene mutations requires a larger study sample size.

In conclusion, the present data revealed a distinct association between EGFR/KRAS mutation status and the ALK rearrangement and predominant subtype, according to the novel IASLC/ATS/ERS LAC scheme. EGFR mutations were significantly associated with the acinar and papillary predominant subtypes, whereas KRAS mutations were significantly associated with the solid predominant subtype and invasive mucinous adenocarcinoma. ALK rearrangements were significantly characterized by the solid predominant subtype with mucin production, the cribriform pattern and a signet-ring cell appearance. The histological subtype proposed by the novel IASLC/ATS/ERS LAC classification may aid the identification of patients with specific genotypes and individualized treatment.

\section{References}

1. Devesa SS, Bray F, Vizcaino AP and Parkin DM: International lung cancer trends by histologic type: Male:Female differences diminishing and adenocarcinoma rates rising. Int J Cancer 117: 294-299, 2005.

2. Jemal A, Bray F, Center MM, Ferlay J, Ward E and Forman D: Global cancer statistics. CA Cancer J Clin 61: 69-90, 2011.

3. Reck M, von Pawel J, Zatloukal P, Ramlau R, Gorbounova V, Hirsh V, Leighl N, Mezger J, Archer V, Moore N and Manegold C: Phase III trial of cisplatin plus gemcitabine with either placebo or bevacizumab as first-line therapy for nonsquamous non-small-cell lung cancer: AVAil. J Clin Oncol 27: 1227-1234, 2009.

4. Jorge SEDC, Kobayashi SS and Costa DB: Epidermal growth factor receptor (EGFR) mutations in lung cancer: Preclinical and clinical data. Braz J Med BiolRes 47: 929-939, 2014.

5. Mitsudomi T, Morita S, Yatabe Y, Negoro S, Okamoto I, Tsurutani J, Seto T, Satouchi M, Tada H, Hirashima T, et al; West Japan Oncology Group: Gefitinib versus cisplatin plus docetaxel in patients with non-small-cell lung cancer harbouring mutations of the epidermal growth factor receptor (WJTOG3405): An open label, randomised phase 3 trial. Lancet Oncol 11: 121-128, 2010.

6. Motoi N, Szoke J, Riely GJ, Seshan VE, Kris MG, Rusch VW, Gerald WL and Travis WD: Lung adenocarcinoma: Modification of the 2004 WHO mixed subtype to include the major histologic subtype suggests correlations between papillary and micropapillary adenocarcinoma subtypes, EGFR mutations and gene expression analysis. Am J Surg Pathol 32: 810-827, 2008.

7. Yatabe Y, Koga T, Mitsudomi T and Takahashi T: CK20 expression, CDX2 expression, K-ras mutation and goblet cell morphology in a subset of lung adenocarcinomas. J Pathol 203: 645-652, 2004.

8. Pao W, Miller V, Zakowski M, Doherty J, Politi K, Sarkaria I, Singh B, Heelan R, Rusch V, Fulton L, et al: EGF receptor gene mutations are common in lung cancers from 'never smokers' and are associated with sensitivity of tumors to gefitinib and erlotinib. Proc Natl Acad Sci USA 101: 13306-13311, 2004.

9. Riely GJ, Marks J and Pao W: KRAS mutations in non-small cell lung cancer. Proc Am Thorac Soc 6: 201-205, 2009.

10. Soda M, Choi YL, Enomoto M, Takada S, Yamashita Y, Ishikawa S, Fujiwara S, Watanabe $\mathrm{H}$, Kurashina K, Hatanaka H, et al: Identification of the transforming EML4-ALK fusion gene in non-small-cell lung cancer. Nature 448: 561-566, 2007.

11. Berge EM, Lu X, Maxson D, Barón AE, Gadgeel SM, Solomon BJ, Doebele RC, Varella-Garcia M and Camidge DR: Clinical benefit from pemetrexed before and after crizotinib exposure and from crizotinib before and after pemetrexed exposure in patients with anaplastic lymphoma kinase-positive non-small-cell lung cancer. Clinical Lung Cancer 14: 636-643, 2013. 
12. Warth A, Muley T, Meister M, Stenzinger A, Thomas M Schirmacher P, Schnabel PA, Budczies J, Hoffmann H and Weichert W: The novel histologic international association for the study of lung cancer/American thoracic society/European respiratory society classification system of lung adenocarcinoma is a stage-independent predictor of survival. J Clin Oncol 30: 1438-1446, 2012.

13. Russell PA, Barnett SA, Walkiewicz M, Wainer Z, Conron M, Wright GM, Gooi J, Knight S, Wynne R, Liew D and John T: Correlation of mutation status and survival with predominant histologic subtype according to the new IASLC/ATS/ERS lung adenocarcinoma classification in stage III (N2) patients. J Thorac Oncol 8: 461-468, 2013

14. Yoshizawa A, Sumiyoshi S, Sonobe M, Kobayashi M, Fujimoto M, Kawakami F, Tsuruyama T, Travis WD, Date $H$ and Haga H: Validation of the IASLC/ATS/ERS lung adenocarcinoma classification for prognosis and association with EGFR and KRAS gene mutations: Analysis of 440 Japanese patients. J Thorac Oncology 8: 52-61, 2013.

15. Travis WD, Brambilla E, Noguchi M, Nicholson AG, Geisinger KR, Yatabe Y, Beer DG, Powell CA, Riely GJ, Van Schil PE, et al: International association for the study of lung cancer/american thoracic society/european respiratory society international multidisciplinary classification of lung adenocarcinoma. J Thorac Oncol 6: 244-285, 2011.

16. Yoshida A, Tsuta K, Nakamura H, Kohno T, Takahashi F, Asamura H, Sekine I, Fukayama M, Shibata T, Furuta K and Tsuda H: Comprehensive histologic analysis of ALK-rearranged lung carcinomas. Am J Surg Pathol 35: 1226-1234, 2011.

17. Jokoji R, Yamasaki T, Minami S, Komuta K, Sakamaki Y, Takeuchi $\mathrm{K}$ and Tsujimoto M: Combination of morphological feature analysis and immunohistochemistry is useful for screening of EML4-ALK-positive lung adenocarcinoma. J Clin Pathol 63: 1066-1070, 2010.

18. Goldstraw P, Crowley J, Chansky K, Giroux DJ, Groome PA, Rami-Porta R, Postmus PE, Rusch V and Sobin L; International Association for the Study of Lung Cancer International Staging Committee; Participating Institutions: The IASLC lung cancer staging project: Proposals for the revision of the TNM stage groupings in the forthcoming (seventh) edition of the TNM classification of malignant tumours. J Thorac Oncol 2: 706-714, 2007.

19. Shimada Y, Saji H, Nomura M, Matsubayashi J, Yoshida K, Kakihana M, Kajiwara N, Ohira T and Ikeda N: Cancer stem cell-related marker expression in lung adenocarcinoma and relevance of histologic subtypes based on IASLC/ATS/ERS classification. Onco Targets Ther 6: 1597-1604, 2013.

20. Kosaka T, Yatabe Y, Onozato R, Kuwano H and Mitsudomi T: Prognostic implication of EGFR, KRAS, and TP53 gene mutations in a large cohort of Japanese patients with surgically treated lung adenocarcinoma. J Thorac Oncol 4: 22-29, 2009.

21. Cai YR, Zhang HQ, Qu Y, Mu J, Zhao D, Zhou LJ, Yan H, Ye JW and Liu Y: Expression of MET and SOX2 genes in non-small cell lung carcinoma with EGFR mutation. Oncol Rep 26: 877-885, 2011
22. Franklin WA, Haney J, Sugita M, Bemis L, Jimeno A and Messersmith WA: KRAS mutation: Comparison of testing methods and tissue sampling techniques in colon cancer. J Mol Diagn 12: 43-50, 2010.

23. Yu DP, Dong YJ, Zhang HQ, Wang JH, Qu Y, Zhou LJ, Su D, Zhang LL, Zhao D and Cai YR: Differential expression of CRKL and AXL genes in lung adenocarcinoma subtypes according to the epidermal growth factor receptor and anaplastic lymphoma kinase gene status. Biomed Rep 2: 481-489, 2014

24. Song Z, Zhu H, Guo Z, Wu W, Sun W and Zhang Y: Correlation of EGFR mutation and predominant histologic subtype according to the new lung adenocarcinoma classification in Chinese patients. Med Oncol 30: 645, 2013.

25. Tsuta K, Kawago M, Inoue E, Yoshida A, Takahashi F, Sakurai H, Watanabe S, Takeuchi M, Furuta K, Asamura H and Tsuda H: The utility of the proposed IASLC/ATS/ERS lung adenocarcinoma subtypes for disease prognosis and correlation of driver gene alterations. Lung cancer 81: 371-376, 2013.

26. Wang J, Dong Y, Cai Y, Zhou L, Wu S, Liu G, Su D, Li X, Qin N, Nong J, et al: Clinicopathologic characteristics of ALK rearrangements in primary lung adenocarcinoma with identified EGFR and KRAS status. J Cancer Res Clin Oncol 140: 453-460, 2014

27. Zhang Y, Sun Y, Pan Y, Li C, Shen L, Li Y, Luo X, Ye T, Wang R, Hu H, et al: Frequency of driver mutations in lung adenocarcinoma from female never-smokers varies with histologic subtypes and age at diagnosis. Clin Cancer Res 18: 1947-1953, 2012 .

28. Kakegawa S, Shimizu K, Sugano M, Miyamae Y, Kaira K, Araki T, Nakano T, Kamiyoshihara M, Kawashima $O$ and Takeyoshi I: Clinicopathological features of lung adenocarcinoma with KRAS mutations. Cancer 117: 4257-4266, 2011.

29. Rekhtman N, Ang DC, Riely GJ, Ladanyi M and Moreira AL: KRAS mutations are associated with solid growth pattern and tumor-infiltrating leukocytes in lung adenocarcinoma. Mod Pathol 26: 1307-1319, 2013.

30. Ignatius SH, Ziogas A and Zell JA: Primary signet-ring carcinoma (SRC) of the lung: A population-based epidemiologic study of 262 cases with comparison to adenocarcinoma of the lung. J Thorac Oncol 5: 420-427, 2010.

31. Gainor JF, Varghese AM, Ou SH, Kabraji S, Awad MM, Katayama R, Pawlak A, Mino-Kenudson M, Yeap BY, Riely GJ et al: ALK rearrangements are mutually exclusive with mutations in EGFR or KRAS: An analysis of 1,683 patients with non-small cell lung cancer. Clin Cancer Res 19: 4273-4281, 2013.

32. Zhang X, Zhang S, Yang X, Yang J, Zhou Q, Yin L, An S, Lin J, Chen S, Xie Z, et al: Fusion of EML4 and ALK is associated with development of lung adenocarcinomas lacking EGFR and KRAS mutations and is correlated with ALK expression. Mol Cancer 9: 188, 2010. 